\title{
Associação entre o bullying e cyberbullying com as alterações de peso em adolescentes escolares de Olinda-PE: Estudo exploratório
}

Association between bullying and cyberbullying with weight changes in school adolescents in

Olinda-PE: Exploratory study

Asociación entre bullying y cyberbullying con cambios de peso en adolescentes escolares de Olinda-

PE: Estudio exploratório

Recebido: 05/10/2021 | Revisado: 15/10/2021 | Aceito: 30/10/2021 | Publicado: 01/11/2021

Letícia Fernanda Serafim Cabral ORCID: https://orcid.org/0000-0001-7590-0917

Universidade de Pernambuco, Brasil

E-mail: leticiafscabral@hotmail.com

Beatriz de Araújo Gusmão

ORCID: https://orcid.org/0000-0002-2182-9649

Universidade de Pernambuco, Brasil

E-mail: beatriz.gusmao@upe.br

Fernanda Ribeiro Barbosa

ORCID: https://orcid.org/0000-0003-3509-250X

Universidade de Pernambuco, Brasil

E-mail: fernandarb00@gmail.com

André Corsino da Fonseca Neto

ORCID: https://orcid.org/0000-0003-1679-6448

Universidade de Pernambuco, Brasil

E-mail: andre.fonsecaneto@upe.br

Raíssa Soares dos Anjos

ORCID: https://orcid.org/0000-0002-4766-4272

Universidade de Pernambuco, Brasil

E-mail: raissa_soares@hotmail.com

Vanessa Rodrigues Monteiro

ORCID: https://orcid.org/0000-0003-1499-3245

Universidade de Pernambuco, Brasil

E-mail: vanessa.monteiro@upe.br

Valdenice Aparecida de Menezes

ORCID: https://orcid.org/0000-0003-4183-3239

Universidade de Pernambuco, Brasil

E-mail: valdmenezes@hotmail.com

Viviane Colares

ORCID: https://orcid.org/0000-0003-2912-2100

Universidade de Pernambuco, Brasil

E-mail: viviane.colares@upe.br

Carolina da Franca

ORCID: https://orcid.org/0000-0002-7365-2806 Universidade de Pernambuco, Brasil

E-mail: carolina.franca@upe.br

Fabiana Godoy

ORCID: https://orcid.org/0000-0002-1946-9605

Universidade de Pernambuco, Brasil

E-mail: fabiana.godoy@upe.br

\begin{abstract}
Resumo
Objetivo: Analisar associação entre o bullying com as alterações de peso em adolescentes. Metodologia: Estudo transversal, participaram os adolescentes da rede estadual de ensino de Olinda, entre 14 a 19 anos e ambos os sexos. O instrumento utilizado foi a PeNSe e a avaliação antropométrica. Resultados: Obtiveram-se que para análise do bullying, os adolescentes do sexo masculino (23,1\%), entre $14-16$ anos de idade $(26,3 \%)$ e obesos $(44 \%)$ eram mais acometidos. Dentre as variáveis analisadas, apenas IMC foi estatisticamente significativo para que sofresse bullying escolar. Em se tratando de cyberbullying, a maioria foi do sexo feminino (9,8\%), tinha entre $17-19$ anos $(11,8 \%)$ e eram obesos $(16 \%)$. Diferentemente do bullying, as variáveis analisadas não foram estatisticamente significativas para a prática do cyberbullying. Discussão: Em relação ao sexo, observou-se no presente estudo uma distribuição homogênea entre os
\end{abstract}


sexos feminino e o masculino, em casos tanto de bullying como de cyberbullying. Em relação a idade, ser mais jovem (14-16 anos), é considerado um fator atenuante como aqueles com sobrepeso/obesidade para que se tornem vítimas de bullying, ao contrário do cyberbullying, que teve sua maior parte de jovens entre 17-19 anos. Conclusão: A prática do bullying causa prejuízos à saúde mental e física desses jovens que estão em uma fase de desenvolvimento.

Palavras-chave: Adolescentes; Índice de massa corporal; Bullying.

\begin{abstract}
Objective: Analyze the association between bullying and weight changes in adolescents. Methodology: Cross-sectional study, involving adolescents from the state education system of Olinda, aged between 14 and 19 years and both genders. The instrument used was PeNSe and anthropometric assessment. Results: For analysis of bullying, male adolescents (23.1\%), between $14-16$ years of age (26.3\%) and obese (44\%) were more affected. Among the variables analyzed, only BMI was statistically significant for suffering school bullying. When it comes to cyberbullying, most were female (9.8\%), aged between 17-19 years (11.8\%) and obese (16\%). Unlike bullying, the analyzed variables were not statistically significant for the practice of cyberbullying. Discussion: Regarding gender, it was observed in the present study a homogeneous distribution between females and males, in cases of both bullying and cyberbullying. Regarding age, being younger (14-16 years) is considered a mitigating factor as those with overweight/obesity to become victims of bullying, unlike cyberbullying, which had mostly young people between 17-19 years old. Conclusion: The practice of bullying damages the mental and physical health of these young people who are in a developmental stage.
\end{abstract}

Keywords: Adolescents; Body mass index; Bullying.

\title{
Resumen
}

Objetivo: Analizar la asociación entre bullying y cambios de peso en adolescentes. Metodología: Estudio transversal, que involucró a adolescentes del sistema educativo estatal de Olinda, con edades entre 14 y 19 años y ambos sexos. El instrumento utilizado fue PeNSe y evaluación antropométrica. Resultados: Para el análisis del bullying, los adolescentes varones $(23,1 \%)$, entre $14-16$ años $(26,3 \%)$ y obesos $(44 \%)$ fueron los más afectados. Entre las variables analizadas, solo el IMC fue estadísticamente significativo para sufrir bullying escolar. En lo que respecta al ciberbullying, la mayoría eran mujeres $(9,8 \%)$, de entre 17 y 19 años (11,8\%) y obesas (16\%). A diferencia del bullying, las variables analizadas no resultaron estadísticamente significativas para la práctica del cyberbullying. Discusión: En cuanto al género, se observó en el presente estudio una distribución homogénea entre mujeres y hombres, tanto en los casos de bullying como en el cyberbullying. En cuanto a la edad, ser más joven (14-16 años) se considera un factor atenuante para que las personas con sobrepeso / obesidad se conviertan en víctimas del acoso escolar, frente al ciberacoso, que tuvo en su mayoría jóvenes de 17 a 19 años. Conclusión: La práctica del bullying daña la salud física y mental de estos jóvenes que se encuentran en una etapa de desarrollo.

Palabras clave: Adolescentes; Indice de Masa Corporal; Bullying.

\section{Introdução}

A adolescência é o período de transição entre a infância e a fase adulta e é marcada por transformações biológicas, fisiológicas e desenvolvimento biopsicossocial. É nessa fase que o adolescente tem contato com inúmeras descobertas e também com instabilidades emocionais, tornando a adolescência um período crítico para o desenvolvimento das competências pessoais e interpessoais, bem como a tomada de decisões. Além disso, é nessa fase que os indivíduos se esforçam para alcançar os objetivos relacionados às expectativas culturais da sociedade em que vive (Senna \& Dessen, 2011).

As expectativas culturais são difundidas através das mídias sociais, publicidade, televisão e também da família e dos amigos. Por isso, há uma imposição de um padrão de beleza aos adolescentes, especialmente no âmbito feminino, que é o sexo mais vulnerável à influência da sociedade e que tem como padrão atrizes e pessoas populares que exibem um corpo magro e esbelto (Manuel, 2004; Gonçalves \& Martinéz, 2014). Esses são aspectos contemporâneos de beleza que contribuem para o surgimento de problemas sociais como é o caso do bullying (Fonseca et al., 2012).

O bullying é definido como comportamento prejudicial intencional, realizado repetidamente, contra um indivíduo que é incapaz de se defender (Olweus, 2013) e se enquadra como violência escolar, sendo bastante discutido por profissionais da saúde e principalmente da educação. Ele compreende várias formas de agressão, proposital e repetida, adotadas por um ou mais indivíduos contra outro ou um grupo em uma relação desigual de poder. Pesquisas apontam a necessidade de priorizar estratégias 
de prevenção do bullying nas instituições de ensino, que são os locais mais comuns de ocorrência e perpetuação (Fonseca et al., 2012).

O bullying ainda pode ser caracterizado como cyberbullying. Essa modalidade de bullying faz uso da tecnologia, através das mídias e redes sociais. Nesse caso, por se tratar de um ambiente virtual, o cyberbullying não ocorre necessariamente no ambiente escolar (Lopes, 2005; Kowalski et al., 2012; Hinduja \& Patchin, 2012). Esse tipo de bullying tem ganhado maior destaque devido ao uso constante de telas e inserção cada vez mais precoce dos indivíduos no ambiente virtual, principalmente no contexto atual de pandemia do Covid-19.

Acredita-se que esses tipos de agressões surgem de problemas sociais, econômicos, culturais e históricos que consequentemente, geram impactos psicológicos negativos que predispõem a presença de percepções constrangedoras, incômodas e estressantes, levando a uma resistência de experiências (Schnaider et al., 2012; Shakoor et al., 2010).

A Organização Mundial da Saúde (OMS) declara que a violência de forma geral é um crescente problema de saúde pública que não pode ser negligenciado (Sousa, 2012). A violência escolar se enquadra nessa crescente e tem feito vítimas que são expostas a intimidações, apelidos cruéis, constrangimentos, acusações injustas, hostilidade, danos físicos, psíquicos ou comportamentais podendo levar à exclusão (Lopes, 2005). Dessa forma, a atenção deve estar voltada para as consequências da violência para os indivíduos, as famílias, as comunidades, bem como a saúde pública dos países, já que os efeitos nocivos da violência na saúde apresentam-se nas suas mais diferentes formas (Sousa, 2012). As pesquisas apontam que na percepção dos adolescentes, a principal causa para ocorrência do bullying é o excesso de peso (Puhl \& Luedicke, 2011). Todavia, também são constatados casos de bullying praticado com adolescentes com baixo peso (Lian et al., 2018).

As várias formas que o bullying assume na atualidade tem envolvido crianças e adolescentes, em especial, essa população que possuem o estereótipo da obesidade, são os mais acometidos por esse tipo de violência. Embora esteja enraizado na cultura brasileira o hábito de dizer que uma criança/adolescente é "obesa porque come muito", deve-se lançar mão de uma reflexão, para que se possa esclarecer alguns acordos sociais e simbólicos expressos neste enunciado. Portanto, culturalmente, existe uma ordem consentida, em torno da qual será construído um universo simbólico em torno da gordura corporal, em que ela já porta um valor negativo nas relações sociais e infelizmente, acaba sendo um critério de classificação e organização (Mattos $e t$ al., 2012). Essa violência repercute como problema de saúde pública e por isso, deve ser conhecida, compreendida e, sobretudo, denunciada e combatida (Sousa, 2012).

Diante do exposto, as principais causas relacionadas à ocorrência do bullying e do cyberbullying se refere às características individuais dos estudantes que os diferenciam em relação ao grupo de pares, da vulnerabilidade de um estudante em relação aos demais (Oliveira et al., 2019), que nesse estudo tem como fator desencadeante da violência as alterações no peso dos adolescentes.

A avaliação do estado nutricional do adolescente consiste na utilização de procedimentos de diagnóstico que possibilitam medir o comportamento, a extensão e os determinantes dos agravos nutricionais. Esse processo de avaliação do estado nutricional dentro de uma população permite a identificação dos fatores causais e estudo das associações entre a condição nutricional e a morbimortalidade e, consequentemente, sobre o crescimento e desenvolvimento físico psicossocial dos adolescentes (Araújo \& Campos, 2008; Barros et al., 2018). O aumento do índice de massa corporal (IMC) é uma problemática que está presente na sociedade devido às mudanças ocorridas nos padrões alimentares nas últimas décadas, como o aumento do consumo de açúcares simples, alimentos industrializados e ingestão insuficiente de frutas e hortaliças, as quais estão diretamente associadas ao ganho de peso dos adolescentes (Enes \& Slater, 2010). Em consequência desse aumento, o bullying e o cyberbullying são comportamentos que podem ser desencadeados por um padrão de beleza imposto na sociedade. Nesse contexto, o objetivo desse estudo será avaliar a associação entre o Índice de Massa Corporal (IMC) e o bullying em adolescentes de 14 a 19 anos de ambos os sexos, da rede estadual de ensino da cidade de Olinda, no estado de Pernambuco. 


\section{Metodologia}

\subsection{Delineamento do estudo}

Trata-se de um estudo transversal e exploratório. Este é oriundo de um projeto maior intitulado: "Saúde bucal e comportamentos de risco à saúde modificáveis na adolescência - acompanhar para prevenir”, no qual se pretendeu analisar as condutas de risco à saúde entre os adolescentes.

No estudo transversal, as observações e mensurações das variáveis são feitas de forma simultânea, correspondendo a uma fotografia do que acontece num dado momento, que é a fase da coleta de dados. As fases de delineamento deste estudo correspondem à seleção da população, que é escolhida por apresentar características que possibilitem a investigação e a verificação simultânea de exposição e doença de cada participante (Pereira, 2015). O projeto exploratório é definido como um instrumento em pequena escala capaz de reproduzir os meios e métodos planejados para uma pesquisa que será encontrada na coleta de dados definitiva (Silva, 2015).

\subsection{População alvo}

Adolescentes na faixa etária de 14 a 19 anos e ambos os sexos, regularmente matriculados na rede estadual de ensino da cidade de Olinda.

\subsection{Local de estudo}

O presente estudo foi realizado no ano de 2018 em Olinda. Este é um município brasileiro localizado na Região Metropolitana do Recife - PE que possui uma extensão territorial de 41, $300 \mathrm{Km}^{2}$. No ano em que a pesquisa foi realizada, a região possuía uma população estimada de 391.835 habitantes, sendo 62.025 adolescentes na faixa etária de 10 a 19 anos, representando cerca de $16 \%$ da população total.

Além disso, o município de Olinda apresentava uma taxa de densidade demográfica de 9.063, ou seja, 58 habitantes por quilômetros quadrados, assim sendo a maior densidade demográfica do estado e a quinta maior do Brasil em 2018 (IBGE, 2018).

\subsection{Seleção da amostra}

Em 2018, o município de Olinda possuía 8.902 alunos regularmente matriculados no ensino médio da rede estadual de ensino, os quais estavam distribuídos em 39 escolas públicas estaduais. Destas, 27 escolas foram consideradas elegíveis para a aplicação do questionário sendo uma de pequeno porte, uma de médio porte e 25 de grande porte (SIEPE, 2018).

Todos os alunos presentes foram convidados a participar da pesquisa. Os Termos de Consentimento Livre e Esclarecido (TCLE) foram fornecidos aos pais ou responsáveis ou aqueles com mais de 18 anos para serem assinados. Além disso, os Termos de Assentimento foram entregues aos adolescentes para que estes assinassem, aceitando participar da pesquisa. Foram entregues aproximadamente 12 mil termos e houve uma devolução de 2.500 termos preenchidos, tendo $91 \%$ de todas as salas de aula e $87 \%$ das escolas envolvidas participado da coleta de dados. A amostra total do estudo maior foi de 2.340 adolescentes, mas para o estudo exploratório se considerou 10\% desse valor, ou seja, 234 alunos oriundos de seis escolas.

\subsection{Critérios de elegibilidade e exclusão}

Os critérios de elegibilidade para compor a amostra foram: estudantes adolescentes de ambos os sexos, na faixa etária de 14 a 19 anos e regularmente matriculados no ensino médio das escolas públicas estaduais de Olinda. Os critérios de exclusão foram: estudantes com alguma deficiência ou disfunção que impossibilite o autopreenchimento do questionário e/ou adolescentes 
gestantes. Caso as questões referentes aos dados sociodemográficos (sexo, idade e IMC) e ao bullying não estiverem respondidas, estas são consideradas perdas.

\subsection{Coleta de dados}

Trata-se de uma pesquisa que utilizou dados coletados nos meses de fevereiro e março do ano de 2018. O questionário foi aplicado em um espaço cedido pela escola, sem a presença dos professores, com todos os alunos presentes no dia da coleta e que concordaram em participar do estudo. A aplicação do questionário foi realizada por duas pesquisadoras, que inicialmente informaram os objetivos da pesquisa para os participantes e deixaram claro que as respostas fornecidas serão mantidas em sigilo e não influenciaram no desempenho escolar, assim como não seriam avaliadas como certas ou erradas que eram apenas utilizadas para fins de pesquisa. Na sequência, após a distribuição, durante a aplicação dos questionários, as pesquisadoras ficaram disponíveis para esclarecimento de dúvidas. Ao término foi recolhido o instrumento e guardado imediatamente para que garantisse o anonimato. $\mathrm{O}$ exame físico foi analisado, contabilizando as medidas necessárias logo após os alunos responderem aos questionários. foi realizado um teste/reteste para avaliar o grau de concordância da avaliação antropométrica entre os pesquisadores.

Esse resultado foi obtido através do coeficiente de Kappa, onde se obteve um Kappa de 0,99 para altura e 0,96 para circunferência da cintura (CC). Foram adotados os pontos de corte propostos por Taylor et al (2000).

\subsection{Instrumento para coleta de dados}

Os dados foram coletados por meio de um instrumento que continham questões do Youth RiskBehavior Survey (YRBS) validado e adaptado no Brasil e da Pesquisa Nacional de Saúde do Escolar (PeNSE) realizada pelo Instituto Brasileiro de Geografia e Estatística (IBGE). O estudo utilizou questões relacionadas à caracterização sociodemográfica do YRBS como: sexo (feminino/masculino), idade (14 a 16 e 17 a 19 anos); escolaridade da mãe (Ensino fundamental completo/ médio completo); religião (Católica/ Evangélica/ Espírita/afro-brasileira/outra) e renda familiar mensal $(\leq 1$ salário mínimo por mês $>1$ salário mínimo por mês). Além das perguntas referentes ao bullying retiradas da PeNSE: nos últimos 12 meses você já sofreu bullying na sua escola? Nos últimos 12 meses você sofreu bullying virtual incluindo e-mail, bate papo, mensagens instantâneas (Whatsapp), sites (Facebook) ou por mensagem de texto? As respostas eram dicotômicas (sim/não).

Já com relação ao exame físico foram analisadas as seguintes variáveis:

- A avaliação do estado nutricional foi realizada através da aferição de peso, que foi contabilizado por meio de uma balança digital Camry, com capacidade máxima de $150 \mathrm{~kg}$, devidamente calibrada para aferição do peso, em que os adolescentes estavam descalços ou com meias, sem objetos, na posição ereta e com os pés afastados; e da altura, que foi medida com os alunos descalços ou com uso de meias, em posição ereta, os calcanhares encostados e olhando para o horizonte, utilizando um estadiômetro portátil Wiso, com capacidade de dois metros.

- Circunferência da cintura, que utilizou uma fita métrica inelástica, marca seca e com blusa levantada e utilizaram entre o ponto médio da crista ilíaca e a última costela, obtendo duas medidas e a média será usada para análise (Logman, 1986; Gorz $\&$ Tribess, 2010).

- IMC, que é calculado através das curvas de crescimento da Organização Mundial de Saúde (OMS) elaborada para crianças e adolescentes conforme idade e sexo e convertido em desvio padrão (DP) da mediana. Para o cálculo do IMC utilizamos a fórmula padrão (peso/altura²) e posteriormente utilizaremos a classificação pelo score $-Z$ da OMS (OMS, 2007), presente na Tabela 1. 
Tabela 1: Classificação do IMC segundo a OMS, 2007.

\begin{tabular}{|l|l|}
\hline CLASSIFICAÇÃO & SCORE-Z \\
\hline Magreza & $<$ score $-z-2$ \\
\hline Eutrofia & $>/=$ score $-z-2 \mathrm{e}</=$ escore $-\mathrm{z}+1$ \\
\hline Sobrepeso & $>/=$ score $-z+1 \mathrm{e}<$ escore $-\mathrm{z}+2$ \\
\hline Obesidade & score $-z+2$ \\
\hline
\end{tabular}

Fonte: OMS (2017).

\subsection{Análise de dados}

O banco de dados foi construído no SPSS versão 18. Para avaliar o perfil dos alunos foi realizado um cálculo das frequências e percentuais e, a construção das respectivas distribuições de frequência. Para comparar os percentuais encontrados nos níveis dos fatores avaliados foi aplicado o teste Qui-quadrado para comparação de proporção. Ainda, serão calculadas as prevalências do bullying escolar e digital sofrido pelos alunos. Para avaliar a associação do perfil dos alunos avaliados com a ocorrência de bullying foram construídas as tabelas de contingência e aplicado o teste Qui-quadrado para independência. Nos casos em que as suposições do teste Qui-quadrado não foram satisfeitas, foi aplicado então o teste Exato de Fisher. A razão de chances (OR, odds ratio) foi calculada com intervalo de confiança de $95 \%$. Todas as conclusões foram tiradas considerando o nível de significância de 5\%.

\subsection{Aspectos éticos}

Este projeto foi desenvolvido de acordo com a Resolução no 466/2012 do Conselho Nacional da Saúde do Ministério da Saúde e foi aprovado pelo Comitê de Ética em pesquisa (N.2.361.780), determinado pelo Sistema Nacional de Informações Sobre Ética em Pesquisa (SISNEP) com a anuência da Secretaria de Educação do Estado de Pernambuco. Os adolescentes maiores e os menores de 18 anos que tiveram sua participação no estudo, respectivamente, assinaram ou tiveram sua autorização para participação do estudo pelos pais ou responsáveis por meio do Termo de Consentimento Livre e Esclarecido e também a autorização dada por esses menores ao assinar o Termo de Assentimento.

Quanto aos riscos e desconfortos, caso o adolescente participante tivesse se sentido constrangido com alguma pergunta do questionário, foi orientado ao próprio que as respostas foram dadas sem a possibilidade de identificação. As medidas aferidas para evitar constrangimentos foram verificadas em local reservado que não permitia a identificação dessas medidas por outros participantes.

\section{Resultados}

A Tabela 1 mostra que a maioria dos pesquisados eram do sexo feminino (64,2\%), estavam na faixa etária de 14 a 16 anos $(60,3 \%)$ e possuíam baixo peso $(55,8 \%)$. Em relação à categoria do IMC, grande parte se enquadrou na faixa considerada não normal (80,3\%). Todas essas variáveis analisadas foram estatisticamente significantes $(0,001,0,002$ e 0,001 , respectivamente) para ocorrência do bullying. 
Tabela 1. Distribuição do perfil dos alunos avaliados.

\begin{tabular}{lccc}
\hline \multicolumn{1}{c}{ Fator avaliado } & N & $\%$ & p-valor $^{\mathbf{1}}$ \\
\hline Sexo & 145 & 64,2 & \\
Feminino & 81 & 35,8 & $<0,001$ \\
Masculino & & & \\
\hline Idade & 141 & 60,3 & 0,002 \\
14 a 16 anos & 93 & 39,7 & \\
17 a 19 anos & & & \\
\hline IMC & 130 & 55,8 & \\
Baixo peso & 46 & 19,7 & $<0,001$ \\
Eutrófico & 32 & 13,7 & \\
Sobrepeso & 25 & 10,7 & \\
Obesidade & & & $<0,001$ \\
\hline IMC_cat & 46 & 19,7 & 80,3 \\
Normal & 187 & & \\
Não normal & & & \\
\hline
\end{tabular}

${ }^{1} \mathrm{p}$-valor do teste Qui-quadrado para comparação de proporção. Fonte: Autores (2021).

A Tabela 2 infere a distribuição da ocorrência de bullying e cyberbullying entre os adolescentes pesquisados. Verificouse que quase um quarto dos alunos já sofreu bullying escolar $(22,8 \%)$ e uma menor parte o cyberbullying $(10,8 \%)$.

Tabela 2. Distribuição do Bullying escolar e cyberbullying entre os alunos avaliados.

\begin{tabular}{|c|c|c|}
\hline \multirow{2}{*}{ Tipos de Bullying } & \multicolumn{2}{|c|}{ Resposta } \\
\hline & Sim & Não \\
\hline Escolar & $52(22,8 \%)$ & $176(77,2 \%)$ \\
\hline Virtual & $25(10,8 \%)$ & $207(89,2 \%)$ \\
\hline
\end{tabular}

Fonte: Autores (2021).

Na Tabela 3 tem-se a distribuição de bullying escolar e virtual segundo o perfil dos pesquisados. Com relação do bullying escolar, a maioria foi do sexo masculino (23,1\%), tinha entre 14 a 16 anos (26,3\%) e eram obesos (44\%). Dentre as variáveis analisadas apenas o IMC foi estatisticamente significativo $(0,025)$ para ocorrência do bullying escolar. No tocante ao bullying virtual ou seja, cyberbullying, a maioria foi do sexo feminino (9,8\%), tinha entre 17 a 19 anos (11,8\%) e eram obesos (16\%). Dentre as variáveis analisadas, nenhuma foi associada estatisticamente significativa $(0,025)$ para ocorrência de cyberbullying . 
Research, Society and Development, v. 10, n. 14, e274101421439, 2021

(CC BY 4.0) | ISSN 2525-3409 | DOI: http://dx.doi.org/10.33448/rsd-v10i14.21439

Tabela 3. Distribuição do Bullying escolar e cyberbullying segundo o perfil dos pesquisados.

\begin{tabular}{|c|c|c|c|c|}
\hline \multirow{2}{*}{ Fator avaliado } & \multicolumn{2}{|c|}{ Bullying escolar } & \multicolumn{2}{|c|}{ Cyberbullying } \\
\hline & Sim & Não & Sim & Não \\
\hline \multicolumn{5}{|l|}{ Sexo } \\
\hline Feminino & $32(22,5 \%)$ & $110(77,5 \%)$ & $14(9,8 \%)$ & $129(90,2 \%)$ \\
\hline Masculino & $18(23,1 \%)$ & $60(76,9 \%)$ & $7(8,7 \%)$ & $73(91,3 \%)$ \\
\hline p-valor & \multicolumn{2}{|c|}{$0,927^{1}$} & \multicolumn{2}{|c|}{$0,799^{1}$} \\
\hline \multicolumn{5}{|l|}{ Idade } \\
\hline 14 a 16 anos & $36(26,3 \%)$ & $101(73,7 \%)$ & $14(10,1 \%)$ & $124(89,9 \%)$ \\
\hline 17 a 19 anos & $16(17,8 \%)$ & $74(82,2 \%)$ & $11(11,8 \%)$ & $82(88,2 \%)$ \\
\hline p-valor & \multicolumn{2}{|c|}{$0,136^{1}$} & \multicolumn{2}{|c|}{$0,686^{1}$} \\
\hline \multicolumn{5}{|l|}{ IMC } \\
\hline Baixo peso & $21(16,8 \%)$ & $104(83,2 \%)$ & $11(8,6 \%)$ & $117(91,4 \%)$ \\
\hline Eutrófico & $12(26,7 \%)$ & $33(73,3 \%)$ & $5(10,9 \%)$ & $41(89,1 \%)$ \\
\hline Sobrepeso & $7(22,6 \%)$ & $24(77,4 \%)$ & $4(12,9 \%)$ & $27(87,1 \%)$ \\
\hline Obesidade & $11(44,0 \%)$ & $14(56,0 \%)$ & $4(16,0 \%)$ & $21(84,0 \%)$ \\
\hline p-valor & \multicolumn{2}{|c|}{$\mathbf{0 , 0 2 5 ^ { 1 }}$} & \multicolumn{2}{|c|}{$0,595^{2}$} \\
\hline \multicolumn{5}{|l|}{ IMC_cat } \\
\hline Normal & $12(26,7 \%)$ & $33(73,3 \%)$ & $5(10,9 \%)$ & $41(89,1 \%)$ \\
\hline Não normal & $39(21,5 \%)$ & $142(78,5 \%)$ & $19(10,3 \%)$ & $165(89,7 \%)$ \\
\hline p-valor & \multicolumn{2}{|c|}{$0,462^{1}$} & \multicolumn{2}{|c|}{$1,000^{2}$} \\
\hline
\end{tabular}

${ }^{1}$ p-valor do teste Qui-quadrado para independência. ${ }^{2}$ p-valor do teste Exato de Fisher.

\section{Discussão}

Fonte: Autores (2021).

Em 2019, a UNESCO divulgou um relatório em que apresenta o bullying e o cyberbullying como atuais e importantes problemas globais. O relatório traz informações acerca de 144 países, indicando o quanto esses tipos de violência estão presentes a nível global (Educational, 2019). Por isso, nota-se que o bullying e o cyberbullying são cada vez mais colocados em evidência, trazendo à tona diversos estudos com diferentes resultados e reflexões a respeito.

Devido à quantidade crescente de estudos relacionados ao tema nos últimos 5 anos, as variações de prevalência nestes já publicados podem ser explicadas pelas diferentes metodologias utilizadas, bem como os critérios adotados para classificação do bullying. Além disso, outra variação pode ser dada através do modelo explicativo do fenômeno, por se tratar de uma construção multifacetada composta por diferentes domínios. Somado a isso, as diferenças na faixa etária e tamanho das amostras dos estudos podem ser considerados fatores explicativos para discrepância entre os estudos (Alexius et al., 2018).

\subsection{Bullying escolar e sexo}

Em 2019, a UNESCO divulgou um relatório em que apresenta o bullying e o cyberbullying como atuais e importantes problemas globais. O relatório traz informações acerca de 144 países, indicando o quanto esses tipos de violência estão presentes 
a nível global (Educational, 2019). Por isso, nota-se que o bullying e o cyberbullying são cada vez mais colocados em evidência, trazendo à tona diversos estudos com diferentes resultados e reflexões a respeito.

Um estudo realizado por Malta et al. (2010) em escolares de capitais brasileiras revela que 69,2\% dos estudantes nunca sofreram bullying, em consonância com o estudo de Rech et al. (2013) que afirmou que apenas 10,2\% dos entrevistados sofriam esse tipo de violência. Por outro lado, outros estudos também trazem uma grande porcentagem de alunos que sofrem bullying, como é o caso do estudo de Bandeira \& Hutz (2012), que relata que 67,5\% entre 465 estudantes já foram vítimas desse tipo de situação. Já o estudo de Moura et al. (2011) refere que quase um quinto dos adolescentes passaram por essa situação, dado semelhante a um estudo realizado na Malásia por Ganapathy et al. (2019).

Essas prevalências variadas do bullying podem ser explicadas por vários fatores, como: diferentes formas de coletas, questionários utilizados, características das amostras e localização (Pigozi \& Machado, 2015). Além disso, o ano em que se foi pesquisado também é um dado muito importante, pois nos dias atuais se tem muito mais acesso ao que é bullying e campanhas de prevenção contra ele.

Além disso, as diversas variações de prevalência nos estudos já publicados podem ser explicadas pelas diferentes metodologias utilizadas, bem como os critérios adotados para classificação do bullying. Além disso, outra variação pode ser dada através do modelo explicativo do fenômeno, por se tratar de uma construção multifacetada composta por diferentes domínios. Somado a isso, as diferenças na faixa etária podem ser consideradas fatores explicativos para discrepância entre os estudos (Alexius et al., 2018).

Em relação ao sexo, observou-se no presente estudo uma distribuição homogênea entre o feminino e o masculino em casos positivos de violência escolar, e não houve associação estatística significante, estando em consonância com o estudo de Alexius et al. (2018). Apesar disso, em outros estudos pode-se notar uma associação entre o bullying e o sexo, e, na maioria, há uma prevalência maior desse tipo de violência em meninas em relação aos meninos (Annerback et al., 2014; Stensland et al., 2014; Liu et al., 2016; Warkentin et. al., 2016; Lian et al., 2018; Russo, 2020).

No entanto, em uma revisão integrativa realizada no ano de 2014 por Pigozi \& Machado (2015), encontrou-se que a vitimização do bullying estava mais associada ao sexo masculino do que ao feminino. Esse estudo cita a diferença cultural na formação e desenvolvimento de meninos e meninas como um fator que sustenta tais comportamentos, já que meninos geralmente têm comportamentos mais agressivos do que as meninas, o que é fortalecido pelo machismo estrutural.

Além disso, um estudo realizado na Malásia, feito por Ganapathy et al. (2019), descobriu que cerca de 1 em cada 5 adolescentes do sexo masculino e $1 \mathrm{em}$ cada 7 adolescentes do sexo feminino foi vítima de bullying. Esse estudo traz que na época, no âmbito global, a prevalência estava entre $8,6 \%$ a 45,2\% entre os homens e 4,8\% a 35,8\% entre as mulheres, fato que pode ser explicado devido às diferenças culturais dentro das sociedades. As diferenças culturais afetam não apenas a prevalência do bullying dentro de uma sociedade, mas também o peso corporal tido como ideal, a percepção de seu peso corporal e sua influência em ser vítima de bullying.

\subsection{Bullying escolar e faixa etária}

Em relação a variável faixa etária, a maioria dos alunos que afirmaram já terem sofrido bullying encontravam-se entre 14-16 anos, ou seja, os mais novos. Os adolescentes mais novos também são considerados por Lian et al. (2018) como um fator que leva à predisposição do indivíduo de sofrer bullying. Além disso, Liu et al. (2016) em seu estudo, também demonstrou que entre os indivíduos mais jovens há uma maior discriminação quando se relaciona com o corpo do adolescente.

O estudo de Ganapathy et al. (2019) também encontrou que alunos de 13 anos tem 2,5 vezes mais chances de sofrerem bullying em comparação aos de 17. Esses autores também encontraram que, na verdade, qualquer idade abaixo desta tinha uma menor probabilidade de passar por esse tipo de violência. 
Dessa forma, o presente estudo corrobora com esses achados, reforçando a ideia de que quanto maior a idade do adolescente, menor a prevalência do bullying. Esse achado pode ser justificado pelo maior desenvolvimento cognitivo e a melhoria das habilidades sociais nos estudantes com maior idade, podendo facilitar a sua socialização com os colegas e elaboração de estratégias mais adequadas de resolução de conflitos (Oliveira et al, 2019; Silva, 2015). Além disso, podemos considerar que o fator da maturidade nesses casos, podem ser algo relacionado, visto que geralmente quanto menor a idade do adolescente, menor compreensão e discernimento ele tem de suas ações.

\subsection{Bullying escolar e imc}

Com relação à frequência de bullying escolar e IMC, o presente estudo mostrou que os menores percentuais de relatos desse tipo de violência estavam entre os indivíduos de baixo peso e de sobrepeso, enquanto que os obesos relataram serem os que mais sofrem, seguidos dos eutróficos. Os dados também mostraram que houve relevância estatística entre essas duas variáveis.

Espera-se, no entanto, que as vítimas do bullying estejam mais presentes entre os adolescentes com baixo peso e o sobrepeso do que os eutróficos, porém, visto que nessa comunidade escolar o grupo de IMC baixo foi o padrão de normalidade da amostra, confirmando que quem sofre mais este tipo de violência são os que fazem parte da minoria, o diferente, essa variação na apresentação do bullying pode ser explicada através dos padrões impostos pela mídia e sociedade, mostrando a importância da compreensão da população estudada para o entendimento do resultado,

De acordo com Warkentin et al. (2016), tem sido frequente o aumento da prevalência de bullying em adolescentes com sobrepeso e obesidade, corroborando com os resultados do presente estudo e do proposto por Alexius et al. (2018), que faz associação entre bullying e obesidade indicando que os adolescentes discriminados por possuírem características do fenótipo obeso tinham maior chance de serem vítimas de bullying. Além disso, os resultados da pesquisa de Liu et al. (2016) mostraram que o risco de sofrer bullying, levemente aumentado entre os estudantes obesos, mas apenas estatisticamente significativo no grupo feminino. Lian et al. (2018) mostraram que adolescentes com baixo peso também tinham um risco maior de ser cronicamente vítimas de bullying do que adolescentes com peso normal, assim como seus pares com sobrepeso e obesidade. No estudo de Lee et al. (2018) também foi observado resultado semelhante.

Em contrapartida, o estudo de Gong et al. (2019) revelou que, de maneira geral, os alunos que se desviaram do status de peso normal (ou seja, adolescentes com baixo peso, sobrepeso ou obesidade) não foram significativamente associados a serem intimidados se comparado com alunos eutróficos. Esse achado é divergente da maioria dos estudos de países ocidentais, que relatam que há uma predisposição dos jovens com sobrepeso e/ou obesos corriam a serem intimidados. Uma possível razão para a discrepância de resultados é o fato de que a China possui proporções relativamente baixas de pessoas com sobrepeso e obesas em comparação com os países ocidentais. Outra possível explicação poderia ser que a estigmatização de pessoas com sobrepeso na China não é tão forte ou generalizada como nas culturas ocidentais.

\subsection{Cyberbullying e sexo}

Em consonância com os dados de bullying, quando avaliamos a prevalência do cyberbullying, a quantidade de meninos e de meninas que foram acometidos com cyberbullying foi semelhante, afirmando que não houve associação significativa, corroborando com estudos anteriores realizados na Itália e Chile (Menin et al. 2021; Cerón et al. 2018). Céron et al. (2018) reforça que isso pode ser devido ao maior número de campanhas positivas que podem vir a prevenir essa diferença.

Em contrapartida, alguns estudos encontraram associação entre o cyberbullying e o sexo, sendo o sexo feminino considerado muito mais propenso a sofrer esse tipo de violência (Morin et al., 2018; Sergentanis et al., 2021; Foody et al., 2019). Além disso, Gong et al. (2019) revelou que jovens obesas eram muito mais propensas a sofrer cyberbullying do que jovens do 
sexo feminino com IMC normal. Para finalizar a pesquisa acerca do assunto, o estudo de Chang et at. (2013), revelou que a prevalência de cyberbullying foi maior no sexo masculino. Outro achado de Chang et al. (2013) é que o cyberbullying se tornou a principal modalidade de bullying entre adolescentes no Taiwan, podendo esses dados estarem relacionados ao aumento no uso da internet e do telefone celular e também da conveniência e anonimato do ambiente cibernético.

Pode-se notar que há uma diferença entre os diversos achados de cyberbullying com sexo, mas que em sua maioria as meninas sofreram mais este tipo de violência. Isso pode estar relacionado à pressão estética que acomete principalmente o sexo feminino, e que foi exacerbada pelo meio digital, colaborando para a amplificação do bullying virtual.

\subsection{Cyberbullying e faixa etária}

Em relação à faixa etária mais acometida pelo cyberbullying, não houve associação significativa entre cyberbullying e idade nesse estudo, apesar de que em dados percentuais, os alunos que têm entre 17 e 19 anos sofriam mais cyberbullying que os que tinham entre 14 e 16 anos. Esse dado corrobora com os dados do estudo de Smith et al. 2008, no qual a população de estudo foi de 92 alunos da cidade de Londres e verificou-se que a tendência de alunos mais velhos terem sofrido cyberbullying alguma vez não era significativa. Ainda sobre o estudo de Smith et al. (2008), os autores afirmaram que quase $80 \%$ dos adolescentes pesquisados nunca sofreram cyberbullying. Esse alto número pode ser explicado pela pesquisa ter sido realizada em uma época em que ainda não havia tanto acesso à internet, e, consequentemente, o bullying virtual era bem menos comum do que nos dias atuais.

Em contrapartida, o estudo de Sergentanis et al. 2021, afirmou que os estudantes de maior idade tendem a sofrer mais bullying virtual, em comparação com os mais jovens. Como achados, ainda se encontra que a medida que a idade aumenta, o bullying escolar diminui, porém o cyberbullying permanece constante quando se associa a idade (Waasdorp \& Bradshaw, 2015). O aumento do percentual da prática do cyberbullying nos adolescentes com maior idade abre espaço para a possibilidade de que, como essa violência é praticada de forma digital e muitas vezes de forma anônima, faz com que o público alvo possa apresentar modificação com relação ao bullying praticado no ambiente escolar, em que os mais velhos podem possuir um status de maior dominância sobre os mais novos.

\subsection{Cyberbullying e imc}

Ao contrário da maioria das descobertas, que sugerem uma associação positiva entre obesidade e vitimização por cyberbullying, nossos achados em relação ao cyberbullying e IMC, evidenciam que essa associação não foi significativa. Corroborando assim, com o estudo realizado por Lee et al. 2018, no qual, não houve associação entre o cyberbullying e IMC. O estudo não traz uma explicação plausível para esse achado e sugere que haja uma exploração mais aprofundada da temática.

Um estudo recente, realizado em sete países da Europa, mostrou que os adolescentes com excesso de peso tinham mais probabilidade de serem vítimas de cyberbullying em comparação aos eutróficos (Sergentanis et al., 2021). Além disso, no estudo de Desmet et al. (2014), mostra que jovens obesos têm 2,5 vezes mais probabilidade de serem vítimas de cyberbullying do que jovens não obesos. Assim, a vitimização do cyberbullying e do bullying foram achados associados à saúde psicossocial prejudicada entre os jovens obesos.

O estudo de Gong et al. (2019) revela que os alunos com baixo peso tinham menos propensão a serem vítimas de cyberbullying do que os obesos. Entretanto, afirma que ainda há uma maior probabilidade dos jovens com baixo peso de sofrerem esse tipo de violência, se comparado com os eutróficos, diferentemente do presente estudo, que relata que os adolescentes com o IMC baixo sofreram menos cyberbullying até mesmo quando comparados aos eutróficos, podendo ser explicado pelo fato de que eles eram o padrão de normalidade da amostra. 
As taxas de prevalência e os grupos afetados pelo bullying e o cyberbullying variam consideravelmente entre os estudos, refletindo a heterogeneidade nas definições desses dois conceitos, população estudada, período de ocorrência da violência, frequência dos eventos de bullying e cyberbullying, bem como características demográficas da amostra de cada estudo.

\section{Conclusão}

No presente estudo, houve associação estatisticamente significante entre o bullying e o IMC, sendo maior para os obesos, seguido dos eutróficos. Em relação a prevalência do bullying e/ou cyberbullying, esteve presente em torno de um quarto da população, não havendo diferença estatisticamente significante entre sexo e idade. Em relação à variável IMC, mais da metade da população estava com baixo peso.

Destaca-se a dificuldade em discutir de forma mais ampla e precisa os resultados do presente estudo em relação à literatura. Isso acontece devido aos diferentes instrumentos e procedimentos metodológicos usados na literatura para medir a variável desfecho que é representada pelo bullying e a variável de exposição que é representada pelo IMC.

\section{Referências}

Andreolli, A. S., \& Triches, R. M. (2019). Insatisfação corporal, bullying e fatores associados em adolescentes. Ciência \& Saúde, 12(3), 33077. https://doi.org/10.15448/1983-652x.2019.3.33077

Annerbäck, E.-M., Wingren, G., Svedin, C., \& Gustafsson, P. (2010). Prevalence and characteristics of child physical abuse in Sweden - findings from a population-based youth survey. Acta Paediatrica, 99(8), 1229-1236. https://doi.org/10.1111/j.1651-2227.2010.01792.x

Alexius, S. L., Mocellin, M. C., Corrêa, E. N., Neves, J. d., Vasconcelos, F. d. A. G. d., \& Corso, A. C. T. (2018). Evidences of the association between individual attributes and bullying: a cross-sectional study with adolescents from Florianópolis, Santa Catarina State, Brazil. Cadernos de Saúde Pública, 34(12).

Araújo, A. C. T., \& Campos, J. A. D. B. (2008). Subsídios para a avaliação do estado nutricional de crianças e adolescentes por meio de indicadores antropométricos. Alim. Nutr, 19(2), 219-225.

Bandeira, C. D. M., \& Hutz, C. S. (2012). Bullying: prevalência, implicações e diferenças entre os gêneros. Psicologia Escolar e Educacional, 16 , 35-44.

Barros, J. P, et al. (2018). Bullying em adolescentes obesos e eutróficos no contexto escolar. Contribuciones A Las Ciencias Sociales, 1, 1-5.

Cerón, M. A. et al (2018). Grooming, cyberbullying and sexting in Chile according of sex and school management or administrative dependency. Revista chilena de pediatria, 89(3), 352-360.

Chang, F.-C., Lee, C.-M., Chiu, C.-H., Hsi, W.-Y., Huang, T.-F., \& Pan, Y.-C. (2013). Relationships Among Cyberbullying, School Bullying, and Mental Health in Taiwanese Adolescents. Journal of School Health, 83(6), 454-462. https://doi.org/10.1111/josh.12050

DeSmet, A., Deforche, B., Hublet, A., Tanghe, A., Stremersch, E., \& De Bourdeaudhuij, I. (2014). Traditional and cyberbullying victimization as correlates of psychosocial distress and barriers to a healthy lifestyle among severely obese adolescents - a matched case-control study on prevalence and results from a crosssectional study. BMC Public Health, 14(1). https://doi.org/10.1186/1471-2458-14-224

Dubowitz, H., \& Bennett, S. (2007). Physical abuse and neglect of children. The Lancet, 369(9576), 1891-1899. https://doi.org/10.1016/s0140-6736(07)60856-

Educational, U. N. (2019). Behind the numbers: Ending school violence and bullying. UNESCO.

Enes, C. C., \& Slater, B. (2010). Obesidade na adolescência e seus principais fatores determinantes. Revista Brasileira de Epidemiologia, 13(1), 163-171. https://doi.org/10.1590/s1415-790x2010000100015

Fonseca, M. H. et al. (2012). Bullying: Forma de violência e exclusão escolar. Motricidade, 8(2), 797-802.

Foody, M. et al. (2019). Friendship quality and gender differences in association with cyberbullying involvement and psychological well-being. Frontiers in psychology, 10, 1723.

Ganapathy, S. S., Tan, L., Sooryanarayana, R., Hashim, M. H., Saminathan, T. A., Ahmad, F. H., Salleh, R., \& Abdul Aziz, N. S. (2019). Body Weight, Body Weight Perception, and Bullying Among Adolescents in Malaysia. Asia Pacific Journal of Public Health, 31(8_suppl), 38S-47S. https://doi.org/10.1177/1010539519879339

Gonçalves, V. O., \& Martínez, J. P. (2014). Imagem corporal de adolescentes: um estudo sobre as relações de gênero e influência da mídia. Comunicação \& Informação, 17(2). https://doi.org/10.5216/31792

Gong, Z., Han, Z., Zhang, H., \& Zhang, G. (2019). Weight Status and School Bullying Experiences in Urban China: The Difference Between Boys and Girls. Journal of Interpersonal Violence, 35(15-16), 2663-2686. https://doi.org/10.1177/0886260519880170 
Gorz, F. B., \& Tribess, S. (2010). Circunferência da cintura ou do abdome: qual utilizar para mensurar a gordura visceral? Revista Brasileira de Nutrição Clínica, 25(3), 233-237.

Hinduja, S., \& Patchin, J. W. (2012). Cyberbullying: Neither an epidemic nor a rarity. European Journal of Developmental Psychology, 9(5), 539-543. https://doi.org/10.1080/17405629.2012.706448

Katzer, C., Fetchenhauer, D., \& Belschak, F. (2009). Cyberbullying in Internet-Chatrooms - Wer sind die Täter? Zeitschrift für Entwicklungspsychologie und Pädagogische Psychologie, 41(1), 33-44. https://doi.org/10.1026/0049-8637.41.1.33

Katzer, C., Fetchenhauer, D., \& Belschak, F. (2009b). Cyberbullying: Who Are the Victims? Journal of Media Psychology, 21(1), 25-36. https://doi.org/10.1027/1864-1105.21.1.25

Kowalski, R. M. (2012). Cyberbullying: Bullying in the Digital Age (2a ed.). John Wiley \& Sons.

Kowalski, R. M. et al. (2012a). Chapter 14 Cyber Bullying Among College Students: evidence from multiple domains of college life. Cutting-Edge Technologies In Higher Education, 5, 293-321.

Lee, B., Jeong, S., \& Roh, M. (2018). Association between body mass index and health outcomes among adolescents: the mediating role of traditional and cyber bullying victimization. BMC Public Health, 18(1). https://doi.org/10.1186/s12889-018-5390-0

Lee, K., Dale, J., Guy, A., \& Wolke, D. (2018). Bullying and negative appearance feedback among adolescents: is it objective or misperceived weight that matters?. Journal of Adolescence, 63, 118-128. http://dx.doi.org/10.1016/j.adolescence.2017.12.008.

Lian, Q., Su, Q., Li, R., Elgar, F. J., Liu, Z., \& Zheng, D. (2018). The association between chronic bullying victimization with weight status and body self-image: a cross-national study in 39 countries. PeerJ, 6, Artigo e4330. https://doi.org/10.7717/peerj.4330

Liu, X., Chen, G., Yan, J., \& Luo, J. (2016). Weight status and bullying behaviors among Chinese school-aged children. Child Abuse \& Neglect, 52, 11-19. https://doi.org/10.1016/j.chiabu.2015.12.010

Malta, D. C. (2010). Bullying nas escolas brasileiras: resultados da Pesquisa Nacional de Saúde do Escolar (PeNSE). Revista Ciência \& Saúde Coletiva, 15.

Manuel, S. P. (2004). Estado nutricional y psicosocial en adolescentes con alteración de la conducta alimentaria [Tese (Doutorado) não publicada]. Universitat Rovira I Virgili.

Mattos, R. S. et al. (2012). Obesidade e bullying na infância e adolescência: o estigma da gordura. Demetra, 7(2), 71-84.

Menin, D. et al (2021). Was that (cyber) bullying? Investigating the operational definitions of bullying and cyberbullying from adolescents' perspective. International journal of clinical and health psychology, 21(2), 100221.

Morin, H. K., Bradshaw, C. P., \& Kush, J. M. (2018). Adjustment outcomes of victims of cyberbullying: The role of personal and contextual factors. Journal of School Psychology, 70, 74-88. https://doi.org/10.1016/j.jsp.2018.07.002

Moura, D. R. d., Cruz, A. C. N., \& Quevedo, L. d. Á. (2011). Prevalência e características de escolares vítimas de bullying. Jornal de Pediatria, 87(1), 19-23. https://doi.org/10.1590/s0021-75572011000100004

Oliveira, W. A. d., Silva, J. L. d., Braga, I. F., Romualdo, C., Brandão Neto, W., Caravita, S. C. S., \& Silva, M. A. I. (2019). Percepções de estudantes sobre bullying e família: um enfoque qualitativo na saúde do escolar. Cadernos Saúde Coletiva, 27(2), 158-165. https://doi.org/10.1590/1414-462×201900020478)

Olweus, D. (2013). School Bullying: Development and Some Important Challenges. Annual Review of Clinical Psychology, 9(1), 751-780. https://doi.org/10.1146/annurev-clinpsy-050212-185516

Pereira, P. F., Faria, F. R. d., Faria, E. R. d., Hermsdorff, H. H. M., Peluzio, M. d. C. G., Franceschini, S. d. C. C., \& Priore, S. E. (2015). Indicadores antropométricos para identificar síndrome metabólica e fenótipo cintura hipertrigliceridêmica: uma comparação entre as três fases da adolescência. Revista Paulista de Pediatria, 33(2), 194-203. https://doi.org/10.1016/j.rpped.2014.10.001

Pigozi, P. L., \& Machado, A. L. (2015). Bullying na adolescência: visão panorâmica no Brasil. Ciência \& Saúde Coletiva, 20(11), 3509-3522. https://doi.org/10.1590/1413-812320152011.05292014

Puhl, R. M., \& Luedicke, J. (2011). Weight-Based Victimization Among Adolescents in the School Setting: Emotional Reactions and Coping Behaviors. Journal of Youth and Adolescence, 4l(1), 27-40. https://doi.org/10.1007/s10964-011-9713-z

Rech, R. R. et al. (2013). Prevalence and characteristics of victims and perpetrators of bullying. Jornal de Pediatria, 89, 164-170.

Russo, L. X. (2020). Associação entre vitimização por bullying e índice de massa corporal em escolares. Cadernos de Saúde Pública, 36(10). https://doi.org/10.1590/0102-311x00182819

Schneider, S. K., O'Donnell, L., Stueve, A., \& Coulter, R. W. S. (2012). Cyberbullying, School Bullying, and Psychological Distress: A Regional Census of High School Students. American Journal of Public Health, 102(1), 171-177. https://doi.org/10.2105/ajph.2011.300308

Senna, S. R. C. M., \& Dessen, M. A. (2011). Contribuições das teorias do desenvolvimento humano para a concepção contemporânea da adolescência. Psicologia: Teoria e Pesquisa, 28(1), 101-108. 
Research, Society and Development, v. 10, n. 14, e274101421439, 2021

(CC BY 4.0) | ISSN 2525-3409 | DOI: http://dx.doi.org/10.33448/rsd-v10i14.21439

Sergentanis, T. N., Bampalitsa, S. D., Theofilou, P., Panagouli, E., Vlachopapadopoulou, E., Michalacos, S., Gryparis, A., Thomaidis, L., Psaltopoulou, T., Tsolia, M., Bacopoulou, F., \& Tsitsika, A. (2021). Cyberbullying and obesity in adolescents: Prevalence and associations in seven european countries of the EU NET ADB survey. Children, 8(3), 235. https://doi.org/10.3390/children8030235

Shakoor, S., Jaffee, S. R., Andreou, P., Bowes, L., Ambler, A. P., Caspi, A., Moffitt, T. E., \& Arseneault, L. (2010). Mothers and Children as Informants of Bullying Victimization: Results from an Epidemiological Cohort of Children. Journal of Abnormal Child Psychology, 39(3), 379-387. https://doi.org/10.1007/s10802-010-9463-5.

Silva, L. H. (2015). Contribuições do projeto piloto à coleta de dados em pesquisas na área de educação. Revista Ibero-americana de Estudos em Educação, $10(1), 225-245$.

Smith, P. K. et al (2008). Cyberbullying: Its nature and impact in secondary school pupils. Journal of Child Psychology and Psychiatry, 49(4), 376-385.

Souza, A. C. R. d. (2018). A relevância e o papel dos médicos-dentistas na detecção e prevenção dos maus tratos a menores [Monografia (Especialização) não publicada]. Universidade do Porto.

Stensland, S. Ø., Thoresen, S., Wentzel-Larsen, T., \& Dyb, G. (2014). Interpersonal violence and overweight in adolescents: The HUNT Study. Scandinavian Journal of Public Health, 43(1), 18-26. https://doi.org/10.1177/1403494814556176

U. S. Department of Health \& Human Services. (2020). International classification of diseases 10th revision (ICD-10) (10a ed.). World Health Organization.

Waasdorp, T. E., \& Bradshaw, C. P. (2015). The overlap between cyberbullying and traditional bullying. Journal of Adolescent Health, 56(5), 483-488. https://doi.org/10.1016/j.jadohealth.2014.12.002.

Warkentin, T., Borghese, M. M., \& Janssen, I. (2016). Associations between weight-related teasing and psychosomatic symptoms by weight status among school-aged youth. Obesity Science \& Practice, 3(1), 44-50. https://doi.org/10.1002/osp4.87 